\title{
The Ramsey Number for Tree Versus Wheel with Odd Order
}

\author{
Yusuf Hafidh and Edy Tri Baskoro* \\ Combinatorial Mathematics Research Group \\ Faculty of Mathematics and Natural Sciences \\ Institut Teknologi Bandung \\ Jalan Ganesa 10 Bandung, Indonesia
}

Emails: yusufhafidh@gmail.com, ebaskoro@math.itb.ac.id

\begin{abstract}
Chen et al. (2004) strongly conjectured that $R\left(T_{n}, W_{m}\right)=2 n-1$ if the maximum degree of $T_{n}$ is small and $m$ is even. Related to the conjecture, it is interesting to know for which tree $T_{n}$, we have $R\left(T_{n}, W_{m}\right)>2 n-1$ for even $m$. In this paper, we find the Ramsey number $R\left(T_{n}, W_{8}\right)$ for tree $T_{n}$ with the maximum degree of $T_{n}$ is at least $n-3$, namely $R\left(T_{n}, W_{8}\right)>2 n-1$ for almost all such tree $T_{n}$. We also prove that if the maximum degree of $T_{n}$ is large, then $R\left(T_{n}, W_{m}\right)$ is a function of both $m$ and $n$. In the end, we refine the conjecture of Chen et al. by giving the condition of small maximum degree.
\end{abstract}

Keywords: Ramsey number, tree, wheel.

\section{Introduction}

Let $G$ be a graph with the vertex set $V(G)$ and edge set $E(G)$. All graphs in this paper are assumed to be finite, undirected, and simple graphs. The order of $G$ is the number of vertices of $G$. The graph $\bar{G}$, the complement of graph $G$, is obtained from the complete graph on $|G|$ vertices by deleting the edges of $G$. Let $U \subseteq V(G), G[U]$ is the induced subgraph of $G$ by $U$; that is the maximal subgraph of $G$ with the vertex set $U$. For $v \in V(G)$ and $U, W \subseteq V(G)$, we denote $N_{U}(v)=\{u \in U: u v \in E(G)\}$, and $E(U, W)$ as the edges between $U$ and $W$. The degree of a vertex $v \in V(G)$ is denoted by $\operatorname{deg}(v)$. The maximum (minimum) degree of all vertices in $G$ is denoted by $\Delta(G)(\delta(G))$, respectively.

Let $K_{n}$ be a complete graph on $n$ vertices and let $C_{n}$ be a cycle on $n$ vertices. Let $T_{n}$ be a tree, namely a connected graph with no cycle, on $n$ vertices. A special tree called a star is denoted by $S_{n}$, namely a tree on $n$ vertices with maximum degree $n-1$. Let $W_{m}$ be a wheel on $m+1$ vertices that consists of a cycle $C_{m}$ with one additional vertex being adjacent to all vertices of $C_{m}$. We use $k G$ to denote the disjoint union of $k$ copies of $G$. By using the same notation in 8 , denote by $S_{n}(l, m)$ a tree of order $n$ obtained from $S_{n-m \times l}$ by subdividing each of $l$ chosen edges $m$ times, and denote by $S_{n}(l)$ a tree of order $n$ obtained from a star $S_{l}$ and $S_{n-l}$ by adding an edge joining their centers.

For two graphs $G$ and $H$, a graph $F$ is called $(G, H)$-good if $F$ contains no $G$ and $\bar{F}$ contains no $H$. Any $(G, H)$-good graph on $n$ vertices is called a $(G, H, n)$-good. The Ramsey number $R(G, H)$ is defined as the smallest positive integer $n$ such that no $(G, H, n)$-good graph exists.

Chvátal and Harary (1972) studied the Ramsey numbers for graphs and established the lower bound: $R(G, H) \geq(\chi(H)-1)(c(G)-1)+1$, where $c(G)$ is the number of vertices of the largest component of $G$, and $\chi(H)$ is the chromatic number of $H$. If $G=T_{n}, H=W_{m}$ and $n \geq m \geq 3$, we obtain $R\left(T_{n}, W_{m}\right) \geq 2 n-1$ for even $m$, and $R\left(T_{n}, W_{m}\right) \geq 3 n-2$ for odd $m$.

\footnotetext{
${ }^{*}$ corresponding author
} 
The computation of the Ramsey number $R\left(T_{n}, W_{m}\right)$ has been extensively investigated. However, the results are still far from satisfied. For stars $S_{n}$, the value of $R\left(S_{n}, W_{m}\right)$ is not always equal to the Chvátal-Harary bound [2, 3, 4, 5, 6], but for trees $T_{n}$ other than a star and $S_{n}(1,2)$, all the known Ramsey numbers $R\left(T_{n}, W_{m}\right)$ are equal to the Chvátal-Harary bound [7, 8, 9, 10, 11, 13.

Y. Chen, Y. Zhang, and K. Zhang (2004) strongly conjectured that $R\left(T_{n}, W_{m}\right)=2 n-1$ if the maximum degree of $T_{n}$ is small and $m$ is even. For a tree $T_{n}$ with large maximum degree and even $m$, the $R\left(T_{n}, W_{m}\right)$ is also unknown in general. In this paper, we shall determine the Ramsey number $R\left(T_{n}, W_{8}\right)$ for all trees $T_{n}$ of order $n$ with the maximum degree of $T_{n}$ is at least $n-3$. We also prove that if the maximum degree of $T_{n}$ is large, then $R\left(T_{n}, W_{m}\right)$ is a function of both $m$ and $n$. In the end, we refine the conjecture by giving the condition of small maximum degree.

\section{The Ramsey Number $R\left(T_{n}, W_{m}\right)$}

Related to the revised conjecture proposed by Chen et al. in [8], it is interesting to know for which tree $T_{n}$ we have $R\left(T_{n}, W_{m}\right)>2 n-1$ for even $m$. The first main result deals with the Ramsey number $R\left(T_{n}, W_{8}\right)$ for all trees $T_{n}$ with $\Delta\left(T_{n}\right) \geq n-3$ other than a star, as stated in Theorem 2.1. In the second main result, we derive a general lower bound for $R\left(T_{n}, W_{m}\right)$ for trees $T_{n}$ with large maximum degree and even $m$. This lower bound is a function of both $m$ and $n$. The third main result discusses the conjecture given by Chen et al., we refine this conjecture by providing the condition of small maximum degree for tree $T_{n}$.

Trees $T_{n}$ with $\Delta\left(T_{n}\right) \geq n-3$ will be isomorphic to either $S_{n}, S_{n}(1,1), S_{n}(1,2), S_{n}(2,1)$, or $S_{n}(3)$.

Theorem 2.1. The Ramsey number $R\left(T_{n}, W_{8}\right)$ for $\Delta\left(T_{n}\right) \geq n-3$ is given below.

- $R\left(S_{n}(1,1), W_{8}\right)=\left\{\begin{array}{ll}2 n & , n \equiv 0(\bmod 2) \\ 2 n+1, & n \equiv 1(\bmod 2)\end{array} \quad ; n \geq 5\right.$

- $R\left(S_{n}(1,2), W_{8}\right)=\left\{\begin{array}{ll}2 n & , n \neq 3(\bmod 4) \\ 2 n+1, & n \equiv 3(\bmod 4)\end{array} \quad ; n \geq 8\right.$

- $R\left(S_{n}(2,1), W_{8}\right)=\left\{\begin{array}{ll}2 n-1, & n \equiv 1(\bmod 2) \\ 2 n & , n \equiv 0(\bmod 2)\end{array} \quad ; n \geq 8\right.$

- $R\left(S_{n}(3), W_{8}\right)=\left\{\begin{array}{l}2 n-1, n \equiv 1(\bmod 2) \\ 2 n, n \equiv 0(\bmod 2)\end{array} \quad ; n \geq 8\right.$

The proof of Theorem 2.1 is given in the last section. For $R\left(S_{n}, W_{8}\right)$, see Theorems 3.1 and 3.2 It has been showed in [8], $R\left(S_{n}(1,1), W_{m}\right)$ is a function of both $m$ and $n$, for even m. Precisely, $R\left(S_{n}(1,1), W_{m}\right) \geq 2 n+m / 2-3$ for even $m$ and $n=k m / 2+3$ for any integer $k \geq 2$. It is not a big surprise that the values of $R\left(S_{n}(1,2), W_{m}\right), R\left(S_{n}(2,1), W_{m}\right)$, and $R\left(S_{n}(3), W_{m}\right)$ depends on both $m$ and $n$.

For even $m \geq 8$, and $n \equiv 4(\bmod m / 2)$, define $G=\bar{H} \cup K_{n-1}$ with $H=\left(\frac{2 n+m-8}{m}\right) K_{m / 2}$. Then, $G$ is a $\left(T, W_{m}, 2 n+m / 2-5\right)$-good graph for any tree $T$ with $\Delta(T) \geq n-3$. Hence we have the following theorem.

Theorem 2.2. For trees $T_{n}$ with $\Delta\left(T_{n}\right)=n-3, n \equiv 4(\bmod m / 2)$ and even $m \geq 8, R\left(T_{n}, W_{m}\right) \geq$ $2 n+m / 2-4$.

Moreover, we could modify the graph $H$ above to obtain a lower bound for the other values of $n$. The modification of $H$ could be done by keeping certain properties such as the graph $H$ is $(n-4)$-regular and order $n-4+m / 2$. The graph $H$ is also an union of some graphs with each has order less than $m$. For instance, if $n \equiv 2(\bmod m / 2)$, we could define $H=K_{m / 2-1, m / 2-1} \cup\left(\frac{2 n-4}{m}\right) K_{m / 2}$. In this case, we can easily verify the following theorem. 
Theorem 2.3. For trees $T_{n}$ with $\Delta\left(T_{n}\right)=n-3, n \equiv 2(\bmod m / 2)$ and even $m \geq 8, R\left(T_{n}, W_{m}\right) \geq$ $2 n+m / 2-4$.

For even $m$ and $1 \leq t \leq m / 2-2$, we can get a lower bound for $R\left(S_{n}(1,2 t), W_{m}\right)$ by defining $G=\bar{H} \cup K_{n-1}$ with $H=\left(\frac{2 n+m-2 t-4}{m}\right) K_{m / 2}$ for $n \equiv t+2(\bmod m / 2)$. Since $G$ is a $\left(S_{n}(1,2 t), W_{m}, 2 n+\right.$ $m / 2-t-3)$-good graph, we have the following theorem.

Theorem 2.4. For $n \equiv t+2(\bmod m / 2)$ and even $m \geq 8, R\left(S_{n}(1,2 t), W_{m}\right) \geq 2 n+m / 2-t-2$.

For $t=1$, Theorem 2.4 gives a better lower bound for $R\left(S_{n}(1,2), W_{m}\right)$ compared to Theorem 2.2

Corollary 2.1. For $n \equiv 3(\bmod m / 2)$ and even $m \geq 8, R\left(S_{n}(1,2 t), W_{m}\right) \geq 2 n+m / 2-3$.

In [7, it is conjectured that for every tree $T_{n}$ other than a star and $n>m$, the Ramsey number $R\left(T_{n}, W_{m}\right)$ is equal to the Chvátal-Harary bound $\left(R\left(T_{n}, W_{m}\right)=2 n-1\right.$ for even $m \geq 6$, and $R\left(T_{n}, W_{m}\right)=3 n-2$ for odd $\left.m \geq 7\right)$. For odd $m$, the conjecture is true for $n \geq \frac{m+1}{2} \geq 3$ [4] and for all ES-Trees which is believed to be all Trees [13. However, the conjecture must be refined for even $m$, see Theorem 2.1 and [8]. In 2004, Chen et al. believed that the conjecture is true for even $m$ if $T_{n}$ has small maximum degree. However they did not specify the condition of "small" maximum degree. In this paper, we provide a refinement of the conjecture by giving the condition of small maximum degree as follows.

Conjecture 2.1. For even $m$ and $n>m \geq 4$,

$$
\Delta\left(T_{n}\right) \leq n-m+2 \quad \Rightarrow \quad R\left(T_{n}, W_{m}\right)=2 n-1
$$

The conjecture is true for $m=4(7])$ and $m=6([9,[10,11)$. We cannot increase the condition of the maximum degree because Theorem 2.4 gives the following corollary.

Corollary 2.2. For $n \equiv 0(\bmod m / 2)$ and even $m \geq 8, \Delta\left(S_{n}(1, m-4)\right)=n-m+3$ and $R\left(S_{n}(1, m-\right.$ 4), $\left.W_{m}\right) \geq 2 n$.

\section{Proof of Theorem 2.1}

We give some known Ramsey number and lemmas we use to prove Theorem 2.1.

Theorem 3.1. [5] $R\left(S_{n}, W_{8}\right)=2 n+2$, for even $n \geq 6$.

Theorem 3.2. [6] $R\left(S_{n}, W_{8}\right)=2 n+1$, for odd $n \geq 5$.

Lemma 3.1. [8] Let $G$ be a graph of order $n \geq 6$ with $\delta(G) \geq n-3$, then $G$ contains $S_{n}(3)$ and $S_{n}(2,1)$.

Lemma 3.2. Let $G$ be a graph of order $2 n, n \geq 8$. If $G$ contains $S_{n}(1,1)$ and $\bar{G}$ contains no $W_{8}$, then $G$ must contain $S_{n}(1,2), S_{n}(2,1)$ and $S_{n}(3)$.

Proof. Let $G$ be a graph satisfying the above assumptions. Let $\left\{u_{0}, u_{1}, \cdots, u_{n-1}\right\}$ be the set of the vertices of $S_{n}(1,1)$ in $G$ with $u_{0}$ as the hub and $u_{0} u_{1}, u_{1} u_{n-1} \in E(G)$. Let $U=\left\{u_{2}, u_{3}, \cdots, u_{n-2}\right\}$ and $W=V(G)-V\left(S_{n}(1,1)\right)=\left\{w_{1}, w_{2}, \cdots, w_{n}\right\}$.

First, we prove that $G$ contains $S_{n}(2,1)$. Assume $G$ contains no $S_{n}(2,1)$ then $E(U, W)=\emptyset$ and $E(G[U])=\emptyset$. If $\delta(G[W]) \geq n-3$ then by Lemma 3.1, $W$ contains a $S_{n}(2,1)$, therefore $\delta(G[W]) \leq n-4$ and $\Delta(\bar{G}[W]) \geq 3$. Let $w_{1} \in W$ with $\left\{w_{2}, w_{3}, w_{4}\right\} \subseteq N \overline{G[W]}\left(w_{1}\right)$. This implies that the subgraph of $\bar{G}$ induced by $\left\{w_{1}, w_{2}, w_{3}, w_{4}, u_{2}, u_{3}, u_{4}, u_{5}, u_{6}\right\}$ contains a $W_{8}$ with the hub $w_{1}$ and $w_{2} u_{2} w_{3} u_{3} w_{4} u_{4} u_{5} u_{6} w_{2}$ as the cycle, a contradiction.

Next we prove that $G$ contains $S_{n}(3)$. Assume $G$ contains no $S_{n}(3)$ then $N\left(u_{1}\right) \subseteq\left\{u_{0}, u_{n-1}\right\}$ and $\left|N_{W}\left(u_{i}\right)\right| \leq 1$ for $i \in[2, n-2]$. Since $E(U, W) \leq n-3$ and $|W|=n \geq 8$, there exist $w_{1}, w_{2}, w_{3}, w_{4}$ with $N_{U}\left(w_{i}\right) \leq 1$ for $i=1,2,3,4$. This means that the subgraph of $\bar{G}$ induced by $\left\{u_{1}, u_{2}, u_{3}, u_{4}, u_{5}, w_{1}, w_{2}, w_{3}, w_{4}\right\}$ contains a $W_{8}$ with $u_{1}$ as the hub, a contradiction. 
Finally we prove that $G$ contains $S_{n}(1,2)$. Assume $G$ contains no $S_{n}(1,2)$, then $N\left(u_{n-1}\right) \subseteq\left\{u_{0}, u_{1}\right\}$ and $\left|N_{U}(w)\right| \leq 1$ for each vertex $w \in W$. If there is a vertex in $U$ with at least 3 neighbors in $W$, say $\left\{w_{1}, w_{2}, w_{3}\right\} \subseteq N_{W}\left(u_{2}\right)$, then the subgraph of $\bar{G}$ induced by $\left\{u_{3}, u_{4}, u_{5}, u_{6}, u_{n-1}, w_{1}, w_{2}, w_{3}, w_{4}\right\}$ contains a $W_{8}$ with $u_{n-1}$ as the hub, a contradiction. If there is no vertex in $U$ with at least 3 neighbors in $W$ then any four vertices $u_{2}, u_{3}, u_{4}, u_{5}$ in $U$ and any four vertices $w_{1}, w_{2}, w_{3}, w_{4}$ in $W$ together with $u_{n-1}$ will induce a $S_{n}(1,2)$ in $\bar{G}$ with $u_{n-1}$ as the hub, a contradiction.

Lemma 3.3. If $G$ be a graph of order $n \geq 9$ with $\delta(G) \geq n-4$ and $\Delta(G) \geq n-3$, then $G$ contains $S_{n}(3)$ and $S_{n}(2,1)$.

Proof. Let $V(G)=\left\{w_{1}, w_{2}, \cdots, w_{n}\right\}$ and $w_{1}$ be the vertex with degree at least $n-3$ and $w_{4}, w_{5}, \cdots, w_{n} \in$ $N\left(w_{1}\right)$. First, we prove that $G$ contains a $S_{n}(3)$. If $w_{2}, w_{3} \notin N\left(w_{1}\right)$ or $w_{2} w_{3} \notin E(G)$, then since $\delta(G) \geq n-4$ and $n \geq 9,\left|N\left(w_{1}\right) \cap N\left(w_{2}\right)\right|+\left|N\left(w_{1}\right) \cap N\left(w_{3}\right)\right| \geq n-5+n-5 \geq n-2$ which implies $N\left(w_{1}\right) \cap N\left(w_{2}\right) \cap N\left(w_{3}\right) \neq \emptyset$, and hence $G$ contains a $S_{n}(3)$. Otherwise, without loss of generality $w_{2} \in N\left(w_{1}\right)$ and $w_{2} w_{3} \in E(G)$. Since $\delta(G) \geq n-4$ and $n \geq 9$, we have $\operatorname{deg}\left(w_{2}\right) \geq 5$ which implies $N\left(w_{1}\right) \cap N\left(w_{2}\right) \neq \emptyset$, and hence $G$ contains a $S_{n}(3)$.

Next, we prove $G$ contains a $S_{n}(2,1)$. Since $\delta(G) \geq n-4$ and $n \geq 9$, we have $\operatorname{deg}\left(w_{i}\right) \geq 5$ for all $i$. Therefore $\left|N\left(w_{1}\right) \cap N\left(w_{2}\right)\right| \geq 3$ and $\left|N\left(w_{1}\right) \cap N\left(w_{3}\right)\right| \geq 3$. It is not hard to see that $G$ contains a $S_{n}(2,1)$.

\section{Proof of Theorem 2.1}

We will divide Theorem 2.1 into smaller theorems and prove each of them separately.

Theorem 3.3. $R\left(S_{n}(1,1), W_{8}\right)=2 n+1$ for odd $n \geq 5$.

Proof. Let us consider the graph $G=\overline{\left(\frac{n+1}{4}\right) K_{4}} \cup K_{n-1}$, for $n \equiv 3(\bmod 4)$ and $G=\overline{K_{3,3} \cup\left(\frac{n-5}{4}\right) K_{4}} \cup$ $K_{n-1}$, for $n \equiv 1(\bmod 4)$. It is obvious that $G$ contains no $S_{n}(1,1)$ and $\bar{G}$ contains no $W_{8}$. Hence, we have that $R\left(S_{n}(1,1), W_{8}\right) \geq 2 n+1$ for odd $n$.

In order to show that $R\left(S_{n}(1,1), W_{8}\right) \leq 2 n+1$ for odd $n \geq 5$, consider any graph $G$ of order $2 n+1$ containing no $S_{n}(1,1)$ and $W_{8} \nsubseteq \bar{G}$. First, we will show that $G$ must contain a $S_{n-1}$. In the case of $n \geq 7$, by Theorem 3.1 we obtain that $R\left(S_{n-1}, W_{8}\right)=2(n-1)+2=2 n$ for odd $n \geq 7$. Since $\bar{G}$ contains no $W_{8}$, then $G$ must contain a $S_{n-1}$.

Now, consider if $n=5$. For a contradiction, assume that $G$ contains no $S_{4}$. Then, it implies that $\Delta(G) \leq 2$, and so $\delta(\bar{G}) \geq 8$. Now, consider the graph $\bar{G}$. Let $v$ be a vertex in $\bar{G}$ and let $A=\left\{v_{1}, v_{2}, \cdots, v_{8}\right\} \subseteq N_{\bar{G}}(v)$. Every vertex in $A$ has at least 8 neighbours di $\bar{G}$. Since there are only 3 vertices in $\bar{G}$ that are not in $A$, then every vertex in $\bar{G}[A]$ have at least 5 neighbours in $\bar{G}[A]$, so $\Delta(\bar{G}[A]) \geq 5$. In [1], Bondy showed that if a graph $G_{1}$ with $n_{1}$ vertices have $\delta\left(G_{1}\right) \geq n_{1} / 2$, then $G_{1}$ contains a cycle of any order less or equal to $n_{1}$ or $G_{1}=K_{n_{1} / 2, n_{1} / 2}$ for even $n_{1}$. Therefore $\bar{G}[A]$ contains a $C_{8}$ or $\bar{G}[A]=K_{4,4}$. Since $K_{4,4}$ contains a $C_{8}$, then $\bar{G}$ contains a $W_{8}$, a contradiction. Hence, $G$ contains a $S_{n-1}($ for $n=5)$.

Let $U=\left\{u_{0}, u_{1}, u_{2}, \cdots, u_{n-2}\right\}$ be the set of vertices of $S_{n-1}$ in $G$ with $u_{0}$ as the center. Let $W=\left\{w_{1}, w_{2}, \cdots, w_{n+2}\right\}$ be the set of all vertices in $V(G)-U$. Since $G$ contains no $S_{n}(1,1)$, then $E(U, W)=\emptyset$. Now, consider the following two cases.

Case 1. $\delta(G[W]) \leq n-3$. Let $\operatorname{deg}_{G[W]}\left(w_{1}\right)=\delta(G[W]) \leq n-3$, since $|W|=n+2$ then $\operatorname{deg}_{\bar{G}[W]}\left(w_{1}\right) \geq 4$. Let $\left\{w_{2}, w_{3}, w_{4}, w_{5}\right\} \subseteq N_{\bar{G}[W]}\left(w_{1}\right)$, Then, the induced subgraph in $\bar{G}$ by $\left\{u_{1}, u_{2}, u_{3}\right.$, $\left.u_{4}, w_{1}, w_{2}, w_{3}, w_{4}, w_{5}\right\}$ will contain a $W_{8}$ with $w_{1}$ as the hub, a contradiction.

Case 2. $\delta(G[W]) \geq n-2$. Let $N_{G}\left(w_{1}\right)=\left\{w_{2}, w_{3}, \cdots, w_{n-1}\right\}$. Since $n \geq 5$ and $\delta(G[W]) \geq n-2$, $w_{n}$ has at least 3 neighbours in $G[W]$, then $w_{n}$ have at least a neighbour in $N_{G}\left(w_{1}\right)$ which makes a $S_{n}(1,1)$ in $G[W]$, a contradiction.

Theorem 3.4. $R\left(S_{n}(1,1), W_{8}\right)=2 n$ for even $n \geq 6$.

Proof. Consider the graph $G=\overline{2 K_{4}} \cup K_{n-1}$ for $n=8$ and $G=\bar{C}_{n} \cup K_{n-1}$ for $n \neq 8$. Then, $G$ contains no $S_{n}(1,1)$ and its complement contains no $W_{8}$. Hence, $R\left(S_{n}(1,1), W_{8}\right) \geq 2 n$.

Now, to show that $R\left(S_{n}(1,1), W_{8}\right) \leq 2 n$ for even $n \geq 6$, consider any graph $G$ of order $2 n$ containing no $S_{n}(1,1)$ and $W_{8} \nsubseteq \nsubseteq \bar{G}$. 
By Theorem 3.2, we obtain that $R\left(S_{n-1}, W_{8}\right)=2(n-1)+1=2 n-1$ for even $n$. Since $\bar{G}$ contains no $W_{8}$, then $G$ contains a $S_{n-1}$. Let $U=\left\{u_{0}, u_{1}, u_{2}, \cdots, u_{n-2}\right\}$ be the set of vertices of $S_{n-1}$ in $G$ with $u_{0}$ as the center. Let $W=\left\{w_{1}, w_{2}, \cdots, w_{n+1}\right\}$ be the set of all vertices in $V(G)-U$. Since $G$ contains no $S_{n}(1,1)$, then $E(U, W)=\emptyset$. Now, consider the following cases.

Case 1. $\delta(G[W]) \leq n-4$. Let $\operatorname{deg}_{G[W]}\left(w_{1}\right)=\delta(G[W]) \leq n-4$. since $|W|=n+1$, then $\operatorname{deg}_{\bar{G}[W]}\left(w_{1}\right) \geq 4$. Let $\left\{w_{2}, w_{3}, w_{4}, w_{5}\right\} \subseteq N_{\bar{G}[W]}\left(w_{1}\right)$, then the induced subgraph in $\bar{G}$ by $\left\{u_{1}, u_{2}, u_{3}, u_{4}, w_{1}, w_{2}, w_{3}, w_{4}, w_{5}\right\}$ will contain a $W_{8}$ with the hub $w_{1}$, a contradiction.

Case 2. $\delta(G[W]) \geq n-3$. If $G[W]$ has a vertex of degree at least $n-2$, say $w_{1}$, and let $N_{G}\left(w_{1}\right)=\left\{w_{2}, w_{3}, \cdots, w_{n-1}\right\}$. Since $n \geq 6$ and $\delta(G[W]) \geq n-3, w_{n}$ has at least 3 neighbors in $G[W]$. Hence, one of neighbors of $w_{n}$ is in $N_{G}\left(w_{1}\right)$ and it induces a $S_{n}(1,1)$ in $G[W]$, a contradiction. Therefore, $G[W]$ must be $(n-3)$-regular. But, this is not possible since the order of $G[W]$ is odd, a contradiction.

Corollary 3.1. Let $G$ be a graph of order $2 n$ containing no $S_{n}(1,1)$ with $n \geq 6$. If $\bar{G}$ contains no $W_{8}$ then $n$ is odd and $G=G_{1} \cup G_{2}$ with $G_{1}$ is a graph of order $n-1$ and $G_{2}$ is a regular graph of degree $n-3$.

Proof. Let $G$ be a graph of order $2 n$ satisfying the above assumption. By Theorem 3.4, $n$ must be odd. Theorem 3.1 states that $R\left(S_{m-1}, W_{8}\right)=2 m$ for odd $m$. Since $\bar{G}$ contains no $W_{8}$ and $|V(G)|=2 n$ then $G$ must contain $S_{n-1}$ by Theorem 3.1. Now, by a similar argument as in the proof of Theorem 3.4, we obtain $G=G_{1} \cup G_{2}$ with $G_{1}$ is a graph of order $n-1$ and $G_{2}$ is a regular graph of degree $n-3$.

Theorem 3.5. $R\left(S_{n}(1,2), W_{8}\right)=R\left(S_{n}(2,1), W_{8}\right)=R\left(S_{n}(3), W_{8}\right)=2 n$ for even $n \geq 8$.

Proof. Consider $G=\overline{\left(\frac{n}{4}\right) K_{4}} \cup K_{n-1}$ for $n \equiv 0(\bmod 4)$ and $G=\overline{K_{3,3} \cup\left(\frac{n-6}{4}\right) K_{4}} \cup K_{n-1}$ for $n \equiv$ $2(\bmod 4)$. Then, $G$ contains no tree $T$ with $\Delta(T) \geq n-3$ and its complement contains no $W_{8}$. Hence, we have $R\left(S_{n}(1,2), W_{8}\right) \geq 2 n, R\left(S_{n}(2,1), W_{8}\right) \geq 2 n$, and $R\left(S_{n}(3), W_{8}\right) \geq 2 n$ for even $n$.

Now, for even $n \geq 8$, let $G$ be a graph of order $2 n$ and assume $\bar{G}$ contains no $W_{8}$. By Theorem 3.4 $G$ contains $S_{n}(1,1)$. By Lemma 3.2. $G$ contains $S_{n}(1,2), S_{n}(2,1)$, and $S_{n}(3)$. Hence $R\left(S_{n}(1,2), W_{8}\right)=$ $R\left(S_{n}(2,1), W_{8}\right)=R\left(S_{n}(3), W_{8}\right)=2 n$ for even $n \geq 8$.

Theorem 3.6. $R\left(S_{n}(1,2), W_{8}\right)=2 n+1$ for $n \geq 11$ and $n \equiv 3(\bmod 4)$.

Proof. If $G=\overline{\left(\frac{n+1}{4}\right) K_{4}} \cup K_{n-1}$, then $G$ contains no $S_{n}(1,2)$ and its complement contains no $W_{8}$. Hence, we have $R\left(S_{n}(1,2), W_{8}\right) \geq 2 n+1$ for $n \equiv 3(\bmod 4)$. Now, for any $n \geq 7$ and $n \equiv 3(\bmod 4)$, let $G$ be a graph of order $2 n+1$ and assume $\bar{G}$ contains no $W_{8}$. By Theorem 3.3 , $G$ contains a $S_{n}(1,1)$. By Lemma $2, G$ contains $S_{n}(1,2)$. Hence, $R\left(S_{n}(1,2), W_{8}\right)=2 n+1$ for $n \geq 7$ and $n \equiv 3(\bmod 4)$.

Theorem 3.7. $R\left(S_{n}(1,2), W_{8}\right)=2 n$ for $n \geq 9$ and $n \equiv 1(\bmod 4)$.

Proof. Let $G=\overline{3 C_{3} \cup\left(\frac{n-9}{4}\right) K_{4}} \cup K_{n-1}$. Then, $G$ contains no $S_{n}(1,2)$ and its complement has no $W_{8}$. Hence, we have $R\left(S_{n}(1,2), W_{8}\right) \geq 2 n$ for $n \equiv 1(\bmod 4)$. Now, for any $n \geq 9$ and $n \equiv 1(\bmod 4)$, assume for a contradiction $G$ as a graph of order $2 n$ containing no $S_{n}(1,2)$ and $\bar{G}$ contains no $W_{8}$.

If $G$ contains $S_{n}(1,1)$, then by Lemma 3.2 $G$ contains a $S_{n}(1,2)$, a contradiction. Therefore $G$ contains no $S_{n}(1,1)$. By Corollary 3.1. we have $G=G_{1} \cup G_{2}$, where $G_{1}$ is a graph of order $n-1$ and $G_{2}$ is a $(n-3)$-regular graph of order $n+1$. Let $V(W)=\left\{w_{1}, w_{2}, \cdots, w_{n+1}\right\}$ be the set of vertices of $G_{2}$. Let $N\left(w_{1}\right)=\left\{w_{2}, w_{3}, \cdots, w_{n-2}\right\}$ and $w_{n-1}, w_{n}, w_{n+1} \notin N\left(w_{1}\right)$. For any $i \in\{n-1, n, n+1\}$, $N\left(w_{i}\right) \cap N\left(w_{1}\right) \neq \emptyset$ because $n \geq 9$. If $\left(w_{i}, w_{j}\right) \in E(G)$ for any $i, j \in\{n-1, n, n+1\}$ then $G[W]$ contains a $S_{n}(1,2)$. Therefore, the subgraph induced by $\left\{w_{1}, w_{n-1}, w_{n}, w_{n+1}\right\}$ is isomorphic to $\overline{K_{4}}$. That means $|W|$ must be a multiple of 4 . But, this is not possible since $|W|=n+1 \equiv 2(\bmod 4)$.

Theorem 3.8. $R\left(S_{n}(2,1), W_{8}\right)=R\left(S_{n}(3), W_{8}\right)=2 n-1$ for odd $n \geq 9$.

Proof. Consider $G=2 K_{n-1}$. Then, $G$ contains no tree of order $n$ and its complement contains no $W_{8}$. Hence we have, $R\left(S_{n}(2,1), W_{8}\right) \geq 2 n-1$ and $R\left(S_{n}(3), W_{8}\right) \geq 2 n-1$. Now for any odd integer $n \geq 9$, let $G$ be a graph of order $2 n-1$ and assume $\bar{G}$ contains no $W_{8}$. We will prove that $G$ contains a $S_{n}(2,1)$ and a $S_{n}(3)$. From Theorem 3.2 contains a $S_{n-2}$. Now, consider the following cases. 
Case 1. $\Delta(G) \geq n-2$. Let $u_{0}$ be a vertex with degree $\Delta(G) \geq n-2$. Let $U=\left\{u_{1}, u_{2}, \cdots, u_{n-2}\right\} \subseteq$ $N\left(u_{0}\right)$, and $W=V(G)-\left(U \cup\left\{u_{0}\right\}\right)=\left\{w_{1}, w_{2}, \cdots, w_{n}\right\}$.

Subcase 1.1. $E(U, W)=\emptyset$. If $\delta(G[W]) \leq n-5$, then $\Delta(\bar{G}[W]) \geq 4$. Let $w_{1}$ be a vertex with degree $\delta(G[W]) \leq n-5$ and $w_{2}, w_{3}, w_{4}, w_{5} \notin N\left(w_{1}\right)$. Then, there will be a $W_{8}$ in $\bar{G}$ with $w_{1}$ as its hub and the vertices $u_{1}, u_{2}, u_{3}, u_{4}, w_{2}, w_{3}, w_{4}$, and $w_{5}$ as its cycle, a contradiction.

Therefore $\delta(G[W]) \geq n-4$. However, $G[W]$ cannot be $(n-4)$-regular since $n$ is odd. Therefore, there is a vertex of degree at least $n-3$ in $G[W]$. Therefore $G[W]$ contains a $S_{n}(3)$ and $S_{n}(2,1)$ by Lemma 3.3

Subcase 1.2. $E(U, W) \neq \emptyset$. Let $u_{1} w_{1} \in E(G), U^{\prime}=U-\left\{u_{1}\right\}$, and $W^{\prime}=W-\left\{w_{1}\right\}$. First, assume $G$ contains no $S_{n}(2,1)$. Since $G$ contains no $S_{n}(2,1)$, then $E\left(G\left[U^{\prime}\right]\right)=\emptyset$ and $E\left(U^{\prime}, W^{\prime}\right)=\emptyset$. If $\delta(G[W]) \geq n-3$, then $G[W]$ contains a $S_{n}(2,1)$ by Lemma 3.1, so $\delta(G[W]) \leq n-4$. Now, consider the following subcases:

(a) If $\left|N_{U}\left(w_{1}\right)\right| \geq 3$, then $N\left(u_{1}\right)=\left\{u_{0}, w_{1}\right\}$, since otherwise $G$ contains a $S_{n}(2,1)$. Since $E\left(U^{\prime}, W^{\prime}\right)=\emptyset$, then we will have a $W_{8}$ in $\bar{G}$ with the hub $u_{1}$ and the cycle formed by the vertices $u_{2}, u_{3}, u_{4}, u_{5}, w_{2}, w_{3}, w_{4}, w_{5}$, a contradiction;

(b) If $\left|N_{U}\left(w_{1}\right)\right|=2$, let $N_{U}\left(w_{1}\right)=\left\{u_{1}, u_{n-2}\right\}$. Then $N\left(u_{1}\right) \subseteq\left\{u_{0}, u_{n-2}, w_{1}\right\}$, since otherwise $G$ contains a $S_{n}(2,1)$. Since $E\left(U^{\prime}, W^{\prime}\right)=\emptyset$, then $\bar{G}$ will contains a $W_{8}$ with $w_{1}$ as the hub and $u_{2}, u_{3}, u_{4}, u_{5}, w_{2}, w_{3}, w_{4}, w_{5}$ forms its cycle, a contradiction;

(c) If $N_{U}\left(w_{1}\right)=\left\{u_{1}\right\}$. Let $w$ be a vertex in $G[W]$ with degree $\delta(G[W]) \leq n-4$ and $w^{1}, w^{2}, w^{3} \notin$ $N_{G[W]}(w)$. Then, again in $\bar{G}$ we will have a $W_{8}$ formed by the vertices $w, w^{1}, w^{2}, w^{3}, u_{2}, u_{3}, u_{4}, u_{5}, u_{6}$ with $w$ as the hub, a contradiction. Therefore, in any case $G$ will contain a $S_{n}(2,1)$.

Next, assume $G$ contains no $S_{n}(3)$, then $N\left(u_{1}\right) \subseteq\left\{u_{0}, w_{1}\right\}$ and $\left|N_{W}\left(u_{i}\right)\right| \leq 1$ for $i=2,3,4, \cdots, n-$ 2. Since $E\left(U^{\prime}, W^{\prime}\right) \leq n-3$ and $|W|=n \geq 9$, there exist four vertices $w_{2}, w_{3}, w_{4}, w_{5}$ such that $N_{U}\left(w_{i}\right) \leq 1$. This implies that $\bar{G}$ contains a $W_{8}$ fomed by $u_{1}, u_{2}, u_{3}, u_{4}, u_{5}, w_{2}, w_{3}, w_{4}, w_{5}$ with $u_{1}$ as the hub, a contradiction. Therefore, $G$ must contain a $S_{n}(3)$.

Case 2. $\Delta(G)=n-3$. Let $u_{0}$ be a vertex with degree $n-3$. Let $U=N\left(u_{0}\right)=\left\{u_{1}, u_{2}, \cdots, u_{n-3}\right\}$, and $W=V(G)-\left(U \cup\left\{u_{0}\right\}\right)=\left\{w_{1}, w_{2}, \cdots, w_{n+1}\right\}$.

Subcase 2.1. $E(U, W)=\emptyset$. If $\delta(G[W]) \leq n-4$, then $\Delta(\bar{G}[W]) \geq 4$. Let $w_{1}$ be a vertex with degree $\Delta(\bar{G}[W])$ and $w_{2}, w_{3}, w_{4}, w_{5} \in N_{\bar{G}[W]}\left(w_{1}\right)$. Since $E(U, W)=\emptyset$, then the vertices $u_{1}, u_{2}, u_{3}, u_{4}, w_{1}, w_{2}, w_{3}, w_{4}, w_{5}$ will form a $W_{8}$ in $\bar{G}$ with the hub $w_{1}$, a contradiction. Therefore $\delta(G[W]) \geq n-3$. Let $w_{2}, w_{3}, \cdots w_{n-2} \in N\left(w_{1}\right)$, then $G\left[W-\left\{w_{1}\right\}\right]$ is a graph of order $n$ with $\delta\left(G\left[W-\left\{w_{1}\right\}\right]\right) \geq n-4$. The graph $G\left[W-\left\{w_{1}\right\}\right]$ cannot be $(n-4)$-regular since $n$ is odd. Therefore, $\Delta\left(G\left[W-\left\{w_{1}\right\}\right]\right)=n-3$. By Lemma 3.3. $G\left[W-\left\{w_{1}\right\}\right]$ contains a $S_{n}(2,1)$ and a $S_{n}(3)$.

Subcase 2.2. $E(U, W) \neq \emptyset$. Let $u_{1} w_{1} \in E(G), U^{\prime}=U-\left\{u_{1}\right\}$, and $W^{\prime}=W-\left\{w_{1}\right\}$. If $\delta\left(G\left[W^{\prime}\right]\right) \geq n-4$ then $G\left[W^{\prime}\right]$ cannot be $(n-4)$-regular since $n$ is odd. Therefore, $\Delta\left(G\left[W^{\prime}\right]\right)=n-3$. But, by Lemma $3.3 G\left[W^{\prime}\right]$ contains a $S_{n}(2,1)$ and a $S_{n}(3)$. Thus, $\delta\left(G\left[W^{\prime}\right]\right) \leq n-5$.

Now, we will prove $G$ contains $S_{n}(2,1)$. Assume to the contrary, $G$ contains no $S_{n}(2,1)$. Since $E(U, W) \neq \emptyset$, let $u_{1} w_{1} \in E(G), U^{\prime}=U-\left\{u_{1}\right\}$, and $W^{\prime}=W-\left\{w_{1}\right\}$. Since $G$ contains no $S_{n}(2,1)$, then $E\left(U^{\prime}, W^{\prime}\right)=\emptyset$. Since $\delta\left(G\left[W^{\prime}\right]\right) \leq n-5$, we have that $\Delta\left(\bar{G}\left[W^{\prime}\right]\right) \geq 4$. Let $w_{2}$ be a vertex of degree $\delta\left(G\left[W^{\prime}\right]\right)$ and $w_{3}, w_{4}, w_{5}, w_{6} \in N_{\bar{G}\left[W^{\prime}\right]}\left(w_{2}\right)$, then the vertices $u_{2}, u_{3}, u_{4}, u_{5}, w_{2}, w_{3}, w_{4}, w_{5}, w_{6}$ will form a $W_{8}$ in $\bar{G}$ with the hub $w_{2}$, a contradiction, and hence $G$ contains $S_{n}(2,1)$.

Next, we prove that $G$ must contain $S_{n}(3)$. Assume to the contrary that $G$ contains no $S_{n}(3)$. Let $a=\max \left\{\left|N_{U}\left(w_{i}\right)\right|: 1 \leq i \leq n+1\right\}$. Let $w_{n+1}$ be a vertex in $W$ with $a$ neighbours in $U$. Then, by a similar argument above, $W_{1}=W-\left\{w_{n+1}\right\}$ has a vertex $w_{1}$ with degree $n-5$. Let $w_{2}, w_{3}, w_{4}, w_{5} \notin N_{G\left[W_{1}\right]}\left(w_{1}\right)$. Consider the following cases.

(a) $a \geq 3$. Let $u_{1}, u_{2}, u_{3} \in N_{U}\left(w_{n+1}\right)$. Since $G$ contains no $S_{n}(3)$ and $N\left(u_{0}\right)=U$, then there will be no edges between $\left\{u_{0}, u_{1}, u_{2}, u_{3}\right\}$ and $\left\{w_{1}, w_{2}, w_{3}, w_{4}, w_{5}\right\}$. Therefore, the vertices $u_{0}, u_{1}, u_{2}, u_{3}, w_{1}, w_{2}$, $w_{3}, w_{4}, w_{5}$ will form a $W_{8} \bar{G}$ with the hub $w_{1}$ and $u_{1} w_{2} u_{2} w_{3} u_{3} w_{4} u_{0} w_{5} u_{1}$ as its cycle, a contradiction. (b) $a=2$. Let $u_{1}, u_{2} \in N_{U}\left(w_{n+1}\right)$. Since $G$ contains no $S_{n}(3)$, then there will be no edges between $\left\{u_{1}, u_{2}\right\}$ and $\left\{w_{1}, w_{2}, w_{3}, w_{4}, w_{5}\right\}$. We could assume $N_{U}\left(w_{1}\right) \subset\left\{u_{n-4}, u_{n-3}\right\}$ since $N_{U}\left(w_{1}\right) \leq a=$ 2. Since $n \geq 9$, we could have $u_{3} \notin N_{U}\left(w_{1}\right)$. Since $G$ contains no $S_{n}(3)$, let $N_{W_{1}}\left(u_{3}\right) \subseteq\left\{w_{2}\right\}$. Therefore, the vertices $u_{1}, u_{2}, u_{3}, u_{4}, w_{1}, w_{2}, w_{3}, w_{4}, w_{5}$ will form a $W_{8}$ in $\bar{G}$ with the hub $w_{1}$ and 
$u_{1} w_{2} u_{2} w_{3} u_{3} w_{4} u_{0} w_{5} u_{1}$ as its cycle, a contradiction.

(c) $a=1$. Let $N_{U}\left(w_{1}\right) \subseteq\left\{u_{n-3}\right\}$, then $u_{2}, u_{3}, u_{4}, u_{5} \notin N_{U}\left(w_{1}\right)$. Since $a=1$, let $N_{U}\left(w_{i}\right) \subseteq\left\{u_{i}\right\}$ for $i=2,3,4,5$. Therefore the vertices $u_{2}, u_{3}, u_{4}, u_{5}, w_{1}, w_{2}, w_{3}, w_{4}, w_{5}$ will form a $W_{8}$ in $\bar{G}$ with the hub $w_{1}$ and $u_{2} w_{3} u_{5} w_{4} u_{3} w_{2} u_{4} w_{5} u_{2}$ as its cycle, a contradiction.

\section{Acknowledgment}

This research was partially supported under WCU Program managed by Institut Teknologi Bandung, Ministry of Research, Technology and Higher Education, Indonesia.

\section{References}

[1] J.A. Bondy, Pancyclic graphs, J. Combin. Theory, Ser. B, 80-84, (1971).

[2] Surahmat and E.T. Baskoro, On the Ramsey Number of a path or a star versus $W_{4}$ or $W_{5}$, Proceeding of the $12^{\text {th }}$ Australian Workshop on Combinatorial Algorithms, Bandung, Indonesia, July 14-17 (2001), 174-179.

[3] Y. J. Chen, Y. Q. Zhang, and K.M. Zhang, The Ramsey numbers of stars versus wheels, European Journal of Combinatorics 25 (2004), 1067-1075.

[4] Hasmawati, E.T. Baskoro, H. Assiyatun, Star-Wheel Ramsey numbers, J. Combin. Math. Combin. Comput. 55 (2005), 123-128.

[5] Y.Q. Zhang, Y.J. Chen, K. Zhang, The Ramsey number for stars of even order versus a wheel of order nine, European Journal of Combinatorics, (2008), 1744-1754.

[6] Y.Q. Zhang, T.C.E. Cheng, Y.J. Chen, The Ramsey number for stars of odd order versus a wheel of order nine, Discrete Mathematics Algorithms and Applications, (2012).

[7] E.T. Baskoro, Surahmat, S.M. Nababan, and M. Miller, On Ramsey number for tree versus wheel of five and six vertices, Graphs and Combin. 18 (2002), 717-721.

[8] Y.J. Chen, Y.Q. Zhang, and K.M. Zhang, The Ramsey number $R\left(T_{n}, W_{6}\right)$ for $\Delta\left(T_{n}\right) \geq n-3$, Applied Mathematics Letters, (2004), 281-285.

[9] Y.J. Chen, Y.Q. Zhang, and K.M. Zhang, The Ramsey number $R\left(T_{n}, W_{6}\right)$ for small $n$, Utilitas Mathematica, 67 (2005).

[10] Y.J. Chen, Y.Q. Zhang, and K.M. Zhang, The Ramsey number $R\left(T_{n}, W_{6}\right)$ for $T n$ without certain deletable sets, Journal of System Science and Complexity, 18 (2005) 95-101.

[11] Y.J. Chen, Y.Q. Zhang, and K.M. Zhang, The Ramsey numbers of trees versus $W_{6}$ or $W_{7}$, European Journal of Combinatorics, 27 (2006) 558-564.

[12] Surahmat, E. T. Baskoro, H.J. Broersma, The Ramsey numbers of large stars-like trees versus large odd wheels, J. Combin. Math. Combin. Comput. 65 (2008), 153-162.

[13] Y. Zhang, H. Broersma, Yaojun Chen, On fan-wheel and tree-wheel Ramsey numbers, Discrete Mathematics, (2016), $2284-2287$. 